\title{
Orthogonal Capability Building Blocks for Flexible AHS Deployment
}

\author{
Philip Koopman \\ Visiting Senior Research Engineer \\ Carnegie Mellon University \\ Institute for Complex Engineered Systems \\ 5000 Forbes Avenue \\ Pittsburgh, PA 15213 USA
}

\author{
Michelle Bayouth \\ Research Engineer \\ Carnegie Mellon University \\ Robotics Institute \\ 5000 Forbes Avenue \\ Pittsburgh, PA 15213 USA
}

\begin{abstract}
Once a baseline level of full automation is possible for an Automated Highway System, there are numerous choices to be made in deploying enhanced capabilities to improve safety, throughput, and travel time. Identifying a set of orthogonal capabilities enables describing multiple deployment paths within a common framework. Sixteen AHS configurations can be formed from a proposed set of orthogonal capabilities including: number of vehicles grouped into an entity (free agent vs. platoon), number of automated lanes (single or multiple), obstacle strategy (exclusion or detection), and system vigilance (trusting or vigilant). This systematic approach reveals a maximally enhanced end-state configuration: a platooned, multi-lane, obstacle detecting, vigilant AHS that could be attained using any of 24 incremental deployment paths. A mapping technique is presented that can assist in risk management by depicting alternative deployment paths and constraints within a single framework.
\end{abstract}

\section{KEY WORDS}

incremental AHS deployment, orthogonal building blocks 


\section{INTRODUCTION}

Reaping the full benefits of an Automated Highway System (AHS) involves a carefully orchestrated deployment of various features to achieve potential improvements in safety, travel time, and highway throughput. Organizing potential feature upgrades into distinct building blocks (capabilities) may aid in understanding and planning the possible alternative deployment paths. This paper proposes four such capability building blocks: the number of vehicles grouped into an entity (free agent vs. platoon), number of automated lanes (single or multiple), obstacle strategy (exclusion or detection), and system vigilance (trusting or vigilant). A mapping technique depicts deployment paths involving these four capabilities.

The issues discussed here involve deploying key capability enhancements starting with a minimal but fully-automated highway system. An early definition for an Automated Highway System (AHS) was a user service that provides fully automated vehicle control on dedicated highway lanes in a manner that is compatible with and evolvable from the present highway system. [Stevens et al., 1996, pg. 44] The "fully automated" portion of this definition refers to so-called "hands-off, feet-off" operation of a vehicle, meaning that the vehicle itself controls the throttle, brake, and steering. More recently, the National Automated Highway System Consortium (NAHSC) has expanded this vision of an AHS to also consider lanes that serve both automated and manually operated vehicles. [NAHSC, 1996, chapter 7] Partially automated systems (e.g., lateral-only or longitudinal-only automated control) seem to be likely stepping stones to an eventual fully automated AHS configuration. [Stevens, 1997a] However, this paper is concerned with AHS deployment at and beyond the crucial stage in which vehicle occupants are freed from the driving task.

A baseline level of automation is defined for current purposes as the ability to perform lateral control (i.e., lane-keeping within a particular travel lane), perform longitudinal control (i.e., maintaining a safe operating speed and avoiding crashes into preceding vehicles), and provide a way to minimize encounters with hazards such as roadway obstacles. Single vehicle lane-keeping has been demonstrated on existing conventional highways in a variety of roadway and environmental conditions, [Pomerleau et al., 1996] as well as on highways modified to provide infrastructure assistance. [Shladover, 1992] Speed-keeping applications have long been available in the form of cruise control, and will serve as the foundation for more advanced systems that regulate vehicle separation distance. Although technical challenges remain in developing these baseline functions, the risks associated with providing baseline automation seem small within the development framework of an entire AHS.

There are several opportunities for deploying enhancements beyond baseline AHS functionality. Vehicles could coordinate maneuvers and spacing in order to facilitate lane changing, merging, and 
optimizing traffic throughput. Additionally, vehicles could automatically react to hazardous conditions such as obstacles on the roadway or unexpected behavior from nearby manually driven vehicles. But, there is no clear consensus on a deployment path for these advanced AHS functions.

Planning for deploying enhanced AHS capabilities involves accounting for uncertainties in the research and development of new technology. Furthermore, it is impractical to instantaneously introduce full automation into all vehicles operating on all highways. In response to these difficulties, several alternative strategies have been proposed for the incremental deployment of an AHS. One strategy is initially to deploy fully automated vehicles on dedicated lanes, but only on certain heavily-used roadway segments equipped with special-purpose AHS guidance infrastructure. [Shladover, 1991] Another strategy introduces AHS capabilities onto mass transit vehicles for use on existing High Occupancy Vehicle (HOV) lanes, at least initially subject to the supervision of a safety driver. [Tsao, 1995a] A third general strategy involves gradually increasing the degree of automation of new and refitted vehicles over time, with both AHS vehicles and manually driven vehicles sharing essentially all interstate highways. [Bayouth et al., 1996][Ward, 1997]

Each strategy for incremental deployment has both strengths and weaknesses that should be discussed in terms of a common framework. This paper presents such a common framework based on four key AHS capabilities. The following sections discuss previous work, describe the four capabilities, and present a mapping tool for representing deployment paths. Additionally, examples are provided for using the deployment map to describe alternative deployment strategies, depict deployment constraints, and illustrate a deployment contingency option.

\section{PREVIOUS WORK}

The NAHSC is sponsoring ongoing technical and architectural development of an AHS. The first phase of the current effort examined a number of potential end-state AHS architectures and identified the strengths and weaknesses of each. [NAHSC, 1996] As a result, two main conceptual candidates emerged from many possible end-state AHS configurations considered:

- "Dedicated Lanes," in which platoons of closely-spaced vehicles operate in AHS-only lanes that have strictly controlled admittance so as to exclude manual vehicles as well as potential obstacles. This concept could provide high throughput and reduced control system complexity by operating in a wellregulated roadway environment. However, there are unresolved issues with respect to achieving a viable critical mass of deployed roadway and vehicle upgrades [Ward 1997], technology and human factors in deploying platoons [Shladover, 1997], and the practicability of excluding all obstacles as well as manually driven vehicles from AHS roadways. 
- "Mixed Traffic," in which autonomous vehicles commingle with manually operated vehicles on largely unmodified existing roadways. This concept has the advantage of being applicable to most existing roadways. However, there are concerns about the difficulty of creating an automated system that can cope with potential roadway obstacles as well as manually operated vehicles driven in an aggressive or erratic manner.

In order to better understand underlying issues, the NAHSC selected several "concept attributes" grouped into five categories for further study [NAHSC, 1996]: complete automation only in dedicated lanes or mixed with manual traffic; driver roles; distribution of intelligence and separation policy; obstacle management; and deployment sequence. While useful for managing the study of various issues, these categories were not completely orthogonal (e.g., dedicated lanes presumed high density traffic, which significantly limited possible driver roles). The sixteen activity areas for the AHS Precursor Systems Analyses [Stevens et al., 1996] also provided a breakdown of AHS concerns, but were not entirely suitable for use as deployment building blocks for similar reasons.

Tsao describes a single evolutionary deployment path in detail, including both technical and nontechnical factors. [Tsao, 1995b] The framework presented here fulfills a different role, identifying twentyfour alternative deployment paths based on differing sequences of capability enhancement introductions, with sixteen distinct end-state AHS possibilities. Furthermore, it provides a way to manage risk by illustrating alternatives available when scheduled capability enhancements are delayed or available sooner than anticipated. Thus, once a particular end-state and deployment sequence is chosen, it is possible to represent and study contingency strategies in advance. This may help reduce AHS deployment risk if there is a delay in the development of technology for implementing some key capability.

\section{ORTHOGONAL CAPABILITIES}

Four key AHS capabilities have been specifically selected for orthogonality. A set of capabilities is considered orthogonal if either the baseline or enhanced capability can be deployed regardless of whether other capabilities have been enhanced. This use of orthogonality is not to say that blends of capability enhancements may not occur; indeed, they can and likely will as phased introduction of cost-optimized vehicles supports elements of different enhancements. What is crucial is that each could be deployed independently if desired, and that such deployment does not create a technical necessity for enhancing other capabilities.

The proposed orthogonal capabilities are:

- The entity size: either autonomous "free agents", or multi-vehicle "platoons" 
- The number of automated lanes which automated traffic can exploit using lane change maneuvers: either a single lane, or multiple lanes

- Obstacle strategy: either obstacle exclusion, or obstacle avoidance

- The amount of system vigilance required for unexpected vehicle actions: either none, or complete

The baseline AHS capabilities are: free agents, single automated lane, obstacle exclusion, and no vigilance. This selection was made because, as will be discussed, these are subsets of enhanced capabilities. "Enhanced" capabilities are platooning, multiple adjacent lanes, obstacle avoidance in addition to obstacle exclusion, and complete vigilance. While a binary approach (either "baseline" or "enhanced") is used in describing these capabilities, it is likely that real deployment situations will not be as clear-cut. Nonetheless, there is significant benefit in presenting clearly defined cases to permit concise comparisons of alternatives.

The approach used can be extended to encompass additional orthogonal key capabilities as they may be identified and understood by the research community (e.g., approaches to improving reliability, approaches to stabilize traffic flow for optimum efficiency). While there are other important design issues to be considered in developing an AHS (e.g., safely engaging and disengaging automated control, reacting to weather conditions), those issues do not seem to be orthogonal to other issues, and instead have approaches that depend at least in part are predetermined by the four key capabilities discussed herein. The following sections detail the baseline and enhanced states of each capability.

\section{Entity Size}

A free agent is a single vehicle that maintains relatively large inter-vehicle spacing (perhaps 20 meters at highway speed). Because of the large spacing, a free agent need not have tight coupling of control algorithms with other vehicles. Electronic communication among free agents is not required to avoid collisions, although it might be used to increase efficiency. [Ren et al., 1994]

In contrast, a platoon is a set of two or more vehicles having small inter-vehicle spacing (on the order of a meter), [Shladover, 1995], and much larger inter-platoon spacing (perhaps 60 meters at highway speed [Varaiya, 1993]). Platoons may permit increased vehicle density in order to increase system throughput. [Kanaris et al., 1997] Because of exacting control and inter-vehicle communication requirements, platoons will probably be composed entirely of automated vehicles. (The term platoon has in the past been used to encompass a free agent [Varaiya et al., 1991], but that usage assumed even single vehicles would be platoon-capable, which need not be the case.) 
A vehicular entity is defined as a grouping of vehicles of size one or larger that acts together as a coherent unit. The concept of an entity encompasses both a free agent and a platoon, and is applicable whenever the number of vehicles acting in tight concert is unimportant. (The term "entity" is introduced by [Kanaris et al., 1997], and helps distinguish when entity size is important from when it is not.) Upon examination, it is apparent that in any given driving situation a platoon taken as a whole entity must have at least as much functionality as a free-agent entity. In particular:

- Free agents, as an entity of size one, must be able to perform baseline AHS functionality including avoiding collisions with preceding entities.

- Platoons, as entities of size two or greater, must be able (as a group) to provide baseline AHS functionality, but in addition must perform coordination to avoid collisions among vehicles within the platoon.

*****FIGURE 1 GOES HERE*****

Thus, as shown in Figure 1, a platoon capability is an enhancement of a baseline free agent capability. Because it is simpler, free agent capability must necessarily precede, or be available simultaneously, with the enhancement of platoon capability. (Consider that even platoon-capable vehicles must be able to operate as a single-vehicle entity when they are on an otherwise empty roadway; commercial vehicles that are specifically dedicated to trailing other vehicles may be an exception.) Technical developments required to progress from free agent capability to platoon capability include precise automated longitudinal control, robust inter-vehicle communication, coordination algorithms to deal with joining and splitting vehicles, and coordinated emergency behavior. [Ioannou, 1997]

\section{Number of Automated Lanes}

A dedicated lane is one in which manually driven vehicles are excluded via mechanisms such as admission gates, barriers, and law enforcement sanctions. This is in contrast to mixed traffic, in which both manual and automatic vehicles must be able to coexist within the same lanes. It may be more difficult to design an automated system for mixed traffic than for dedicated lanes because of the complexities of performing lane changes in the presence of potentially uncooperative manual drivers. However, a more useful distinction for the purposes of defining orthogonal capabilities is not whether the lanes are dedicated to automated vehicles only, but rather whether an entity operates on a single lane only, or whether it can change among multiple lanes. (The issue of manual driver behavior is not presented as a separate capability building block, but rather is considered when defining this and other capabilities.)

Consider a single lane, in which vehicles enter automated operation either at a single entry gate or by being "turned on" by the driver once positioned in a particular lane. While more than one lane may be 
present, an automated vehicle does not change lanes. In this situation, it is sufficient for any particular entity operating on that single lane to simply lane-keep, maintain speed, and avoid colliding with the preceding entity (obstacle handling is orthogonal to single lane capabilities). Whether or not the entity in front of an automated entity is manually or automatically operated does not change the base functionality required, because even a preceding automated entity might need to perform a sudden stop, decelerate quickly as the result of a collision, or suffer breakdown. Lateral inter-vehicle collisions are impossible if lane barriers are provided. (If barriers are not used, collisions may occur if an automated vehicle experiences a failure or a human driver makes a mistake. But, in the worst case, the presence of manual drivers may increase, rather than solely determine, the chance of longitudinal collision.)

Now consider multiple lanes, in which automated vehicles are permitted to change among a set of adjacent lanes. Longitudinal spacing and speed control must be provided as in the single lane case. Additionally, the possibility of lane change maneuvers requires lateral sensing and possibly coordination (e.g., via turn signals or electronic communication) to avoid lateral collisions and "cutting off" other vehicles. Dangerous lane changes or even collisions may take place due to equipment failures, environmental factors such as strong lateral wind gusts over an icy patch, inappropriate obstacle avoidance reactions, or manual driver aggressiveness. Similar to the single lane case, the presence of manual drivers might increase, but does not solely determine, the probability of a collision.

\section{*****FIGURE 2 GOES HERE*****}

Figure 2 illustrates that baseline Single Lane operating capability is a subset of Multiple Lane capability, and must necessarily precede, or be provided simultaneously with, Multiple Lane capability in any deployment path. The presence of manual drivers is not a discriminator in terms of requiring a capability to safely perform lane changing operations and avoid lateral collisions; it changes the probability of an incident (and perhaps whether a particular system configuration is safe enough to be viable for deployment), but not whether an incident can occur. Technology development required to implement multiple lanes might include side- and rear-looking sensors as well as lane changing algorithms that can anticipate and react to concurrent acceleration by multiple vehicles.

\section{Obstacle Strategy}

In order to achieve autonomous operation, entities must not collide with potential obstacles, including objects and disabled vehicles on the roadway. This goal can be achieved by either eliminating the possibility of obstacles being on the roadway, or avoiding collisions when obstacles are detected. 
An obstacle exclusion capability attempts to eliminate all ways in which obstacles can gain access to or be deposited on the roadway. Ways of potentially performing exclusion include: use of physical barriers, inspection of vehicles for loose loads, requiring AHS vehicles to be so reliable that they essentially never break down on the roadway, performing frequent debris removal, and stringent law enforcement activity. [NAHSC, 1996] While it is clear that no obstacle exclusion system can be perfect, the intent is to make incidents and accidents due to obstacles so rare as to be an acceptably low safety risk. Whether that can be accomplished effectively enough to be viable remains uncertain. Civil infrastructure such as encaged roadways may exclude many obstacles and is straightforward to implement, if perhaps expensive.

Eliminating hazards such as dropped vehicle components, animals that gain entry at vehicle access points, and sabotage may prove challenging.

In an obstacle detection strategy, the AHS detects obstacles so that entities may perform obstacleavoidance maneuvers consisting of either braking, lane changing, or both. Examples of obstacles that may need to be detected include not only disabled vehicles and fallen rock, but also people, animals, fallen cargo, pavement buckles, sink holes, large potholes, blown-down highway signs, and possibly even washed-out bridges. Creating obstacle detection sophisticated enough to permit evading or removing animals and difficult-to-sense but dangerous objects while minimizing false alarms could prove difficult.

\section{*****FIGURE 3 GOES HERE*****}

Exclusion is designated as the baseline capability in Figure 3 for two reasons: it is already in place on limited access highways in a limited form (involving varying combinations of access restrictions, fencing, and state vehicle safety inspections), and it is necessary to minimize lane blockages and other traffic disruptions even with obstacle detection. Obstacle detection is an enhanced capability that may be necessary for a viable AHS if exclusion cannot yield a required level of safety at a low enough cost.

\section{System Viqilance}

The preceding discussion has argued that the only difference between mixed traffic and AHS-only traffic is that the rate of human driver errors is (presumably) more frequent than the rate of control failures for an affordable automated system. This higher error frequency could mean that potentially hazardous events would happen more often in a mixed traffic situation, but does not seem to fundamentally change the types of hazardous events that can occur and lead to collisions.

Inter-entity collisions can be avoided by either a policy of trust or vigilance. In a baseline trusting system, an entity must keep from running into the preceding entity. Trailing entities are trusted to do the same. Additionally, each entity must stay within its lane, and entities changing lanes within a multiple 
lane system are responsible for yielding to entities staying within a lane. This trust may be violated by equipment failure, environmental conditions, or human error. While a trusting system might well be at least as safe as current manual driving, it must be recognized that no system will be $100 \%$ dependable, nor will it be guaranteed free from abnormal environmental conditions. Therefore, no AHS can be $100 \%$ trustworthy in terms of freedom from aberrant vehicle behavior. However, some AHS configurations might be trustworthy enough to be considered acceptably safe.

In a vigilant system, an entity must not only attempt to prevent forward impacts, but is enhanced to take corrective action for potential rear impacts, because it does not trust the trailing entities. Similarly, it must attempt to evade lateral impacts because it does not trust neighboring entities in the absence of lane barriers.

\section{*****FIGURE 4 GOES HERE*****}

As Figure 4 shows, vigilant systems must not only have the baseline capability of trusting systems to avoid colliding with other entities, but enhancements to evade being hit as well. Thus the problem of dealing with potentially erratic or aggressive manual driving errors is not whether manual vehicles are included in the system per se, but whether or not any particular entity can trust other entities with respect to unsafe behavior. (This distinction is important, because failed vehicle controls or environmental abnormalities can lead to a breach of trust, and would not be addressed if this area of concern were simply limited to the presence of manual drivers.) It is likely that an AHS will experience failure or environmental extremes on occasion, so the issue of whether a trusted system is safe enough must be based on the acceptable frequency of accidents from all sources, both man and machine. A vigilant system requires information about trailing as well as preceding entities, and control algorithms that detect and react to breaches of trust.

\section{DEPLOYMENT MAP}

Given that it is not currently possible to forecast when any given capability enhancement will be ready for deployment, it seems unlikely that all enhancements will be ready to deploy simultaneously. The first fully automated deployed system might be the baseline set of capabilities, which could be the simplest compared to a system requiring enhanced capabilities. But, an initial deployment might instead require a certain minimum set of capability enhancements to attain viability in the face of safety, social, and other constraints. Thereafter, other capability enhancements can be added as they become feasible, desirable, and cost-effective. The following discussion presents a map that concisely represents all incremental deployment paths involving the four capabilities discussed. 
The actual AHS deployment sequence and desired end-state will be significantly influenced by political, market, environmental, legal, and economic factors. [Stevens, 1997b][Underwood, 1990] The deployment sequence framework presented here can be used to illustrate the limitations imposed by these nontechnical constraints, and thus to represent both technical and non-technical constraints to deployment in a single graphical framework.

\section{A Map for Enhanced Capability Deployment}

In order to construct a deployment map, each capability is assigned a binary value, with a zero indicating that that element is in the simpler, baseline state, and a value of one indicating that the element is in the enhanced capability state. (The possibility of blended or partial technology availability is recognized, but binary representations are used herein for the sake of simplicity to facilitate understanding.)

*****FIGURE 5 GOES HERE*****

*****Table I GOES HERE*****

Figure 5 shows the value assignments for the orthogonal categories discussed in the preceding sections. Given that binary values are assigned to each capability, then various deployment stages can be represented by multi-bit binary numbers, wherein each bit represents whether a particular capability has been enhanced. For the four-element technology set used, this results in a 4-bit binary number representation of all possible major deployment states (Table I). As an example to interpreting Table I, the value 0110 has the leading "0" indicating "Free Agent", the first 1 (the second bit) indicating "Multiple Lanes", the second 1 (the third bit) indicating "Obstacle Detection", and the second 0 (the fourth bit) indicating "Trusting".

*****FIGURE 6 GOES HERE*****

Using the binary numbers of Table I, all sixteen different AHS configurations can be represented in a single picture. The left half of Figure 6 shows a special arrangement of these configurations in the format used for Karnaugh maps (K-maps). [Fletcher, 1980] K-maps are a digital logic design optimization tool; however here the format of the K-map is all that is being used, not the logic optimization methodology.

A special property of a K-map arrangement is that all squares which are horizontally or vertically adjacent differ by exactly one bit in value. In order to accomplish this, edge square adjacency is considered to "wrap around" the map either horizontally or vertically as appropriate. In actuality, a Kmap is a two-dimensional representation of a torus, as shown in the right half of Figure 6. As an example 
of this adjacency property on the two-dimensional map, square 0000 is above square 0001 , to the left of square 0100 , to the "right" of square 1000, and "below" square 0010.

\section{Use of the Deployment Map}

The use of this map in the context of designing an AHS is to plot deployment paths as sequences of introduction for orthogonal capability enhancements. Square 0000 on the map represents baseline automated functionality, with no capability enhancements deployed. A baseline AHS starts, therefore, in square 0000 and evolves by incrementally introducing technologies, represented here by changing one bit from " 0 " to " 1 " at each technology introduction. Each bit change corresponds to a one-square move, either horizontally or vertically, on the deployment map. After all four technology components have been introduced, the AHS is in square 1111, a maximally enhanced capability configuration.

It might be that not all four capability enhancements will be desired (or feasible) for an end-state AHS. Similarly, it might be that a baseline AHS is not acceptably safe to merit deployment. However, it is instructive to assume for the purposes of discussion that state 1111 is the desired end-state, and state 0000 the beginning state. (Thus, this discussion does not encompass how to get to a baseline AHS, but rather how to evolve from baseline automated operation to a more comprehensive AHS system.)

\section{*****FIGURE 7 GOES HERE*****}

Using the K-map in Figure 7, an AHS could be deployed using any sequence of introduction of enhancements, traversing the grid from 0000 to 1111 in four steps. At each step, which capability is enhanced next determines the direction of a move along the map. Two example deployment paths are shown for illustrative purposes. Because Figure 7 depicts a two-dimensional flattening of the threedimensional torus, one arrow appears longer than others, but this is solely an artifact of the order in which different technologies have been labeled on the map.

- Deployment sequence A: This sequence assumes that Multiple Lane capability will occur first, followed by Platooning, Obstacle Avoidance, and finally Vigilance. This is an extension of the dedicated lane evolutionary deployment described in [Tsao, 1995b].

- Deployment sequence B: This deployment sequence envisions Vigilance occurring first, followed by Multiple Lane capability, followed by Obstacle Avoidance, followed by Platooning.

These sample sequences represent two possible deployment paths. What is most notable is that both the Dedicated Lane concept and the Mixed Traffic concept being considered by NAHSC stop short of 
enhancing all four capabilities; thus, there are more capable system configurations possible than those alternatives currently being considered.

\section{$* * * * *$ FIGURE 8 GOES HERE*****}

In addition to representing alternate deployment strategies, a deployment map may be used to represent contingency plans. For example, Figure 8 assumes a particular planned deployment path for the Mixed Traffic concept. However, an alternate deployment path is shown for the contingency that multiple lane technology (0001 to 0101) proves more difficult than expected, and obstacle avoidance capabilities are instead fielded first (0001 to 0011).

Because there are four capabilities, there are 4 factorial, or 24, possible deployment sequences that move from the most simplistic to the most technically comprehensive AHS, assuming that no capabilities are removed after being introduced. The K-map representation permits graphically representation all 24 possible paths.

In addition to graphically representing potential technology deployment sequences, the map in Figures 7 and 8 can also be used as a basis for setting deployment constraints. Rows, columns, and individual boxes can be declared off-limits due to liability constraints, economic constraints, market factors, and socio-political factors. This constraining of deployment paths designates those regions where concurrent multi-technology deployments might required to "jump over" a forbidden region, or a different sequence might be required to avoid a proscribed square.

Figure 8 depicts a hypothetical example constraint that Trusting Multiple Lane systems might be undesirable because of concerns about lateral collisions (requiring enhancement of Vigilance in Multiple Lane configurations), blocking out squares 0100, 0110, 1100, and 1110 from consideration. The K-map approach, therefore, enables the AHS developers to represent and potentially minimize programmatic risk by blocking off paths that are infeasible due to technical and non-technical reasons, and then to explore and develop those paths that are left.

\section{CONCLUSIONS}

A taxonomy of potential AHS configurations can be defined, and exploration of potential deployment paths can be accomplished by defining a set of orthogonal capabilities such as: entity size, number of automated lanes, obstacle strategy, and system vigilance. By defining baseline and enhanced levels of development for each capability a binary representation of each possible deployment combination can be created and depicted on a K-map. A significant finding is that enhanced system configurations exist 
beyond what has been envisioned to date by NAHSC: both the Dedicated Lane concept and the Mixed Traffic concept stop short of a concept that combines the capabilities of both.

The AHS decomposition presented here is a representative way to approach, bound, and manage the problem of AHS deployment. In addition to the actual decomposition into four capability building blocks presented, this paper introduces a methodology and visualization tool for reasoning about deployment sequences that can be used for other orthogonal capability sets that might be proposed in the future. It not only depicts a comprehensive yet small set of deployment paths, but also can be used to depict both technical and non-technical constraints on selecting viable deployment paths and contingency plans. Additionally, the combination of an orthogonal decomposition with a deployment map provides a framework within which critical capabilities can be clearly distinguished and emphasized for systematic development.

\section{ACKNOWLEDGMENTS}

This work was sponsored by USDOT under Cooperative Agreement Number DTFH61-94-X-00001 as part of the National Automated Highway System Consortium. 


\section{REFERENCES}

Bayouth, M. and Thorpe, C., "An AHS Concept Based on an Autonomous Vehicle Architecture," Proceedings of The Third World Congress on Intelligent Transportation Systems, Orlando Florida, 1996.

Fletcher, W., An Engineering Approach to Digital Design, Englewood Cliffs, N.J.: Prentice Hall, 1980.

Ioannou, P., “Control and Sensor Requirements and Issues in AHS.” In: (Ioannou, P., ed.) Automated Highway Systems, New York: Plenum Press, 195-212, 1997.

Kanaris, A., Iaonnou, P., and Ho, F.S., "Spacing and Capacity Evaluations for Different AHS Concepts." In: (Ioannou, P., ed.) Automated Highway Systems, New York: Plenum Press, 125-171, 1997.

NAHSC, "NAHSC Milestone 2 Report: Task C2 Downselect System Configurations and Workshop \#3," Concept Team Draft dated December 12, 1996. National Automated Highway System Consortium report to be submitted the US Department of Transportation in 1997 as a formal program deliverable.

Pomerleau, D., and Jochem, T. "Rapidly Adapting Machine Vision for Automated Vehicle Steering," IEEE Expert, 11(2), 19-27, 1996.

Ren, W. and Green, D., "Continuous platooning: a new evolutionary and operating concept for automated highway systems," Proceedings of the American Control Conference, Baltimore, 21-25, 1994.

Shladover, S.E., "Potential Freeway Capacity Effects of Advanced Vehicle Control Systems," Proceedings of the 2nd International Conference on Applications of Advanced Technologies in Transportation Engineering, 213-217, 1991.

Shladover, S.E., “The California PATH Program: a state approach to IVHS research,” Proceedings 1992 International Congress on Transportation Electronics, Dearborn Michigan, 329-338, 1992.

Shladover, S.E., "Highway Automation Using Platoons," Proceedings of the 1995 Annual Meeting of ITS America, Washington D.C., 51-60, 1995.

Shladover, S.E., "Reasons for Operating AHS Vehicles in Platoons.” In: (Ioannou, P., ed.) Automated Highway Systems, New York: Plenum Press, 11-27, 1997.

Stevens, W., (1997a) “Evolution to an automated highway system.” In: (Ioannou, P., ed.) Automated Highway Systems, New York: Plenum Press, 109-124, 1997. 
Stevens, W., (1997b) "Societal and institutional aspects of AHS deployment.” In: (Ioannou, P., ed.) Automated Highway Systems, New York: Plenum Press, 335-348, 1997.

Stevens, W.B., Harding, J.A., Lay, R. \& McHale, G.M., Precursor Systems Analyses of Automated Highway Systems: Summary of Assessment Findings, Report FHWA-RD-96-071, McLean Virginia: Federal Highway Administration, 1996.

Tsao, J., (1995a) "Constraints on Initial AHS Deployment and the Concept Definition of a Shuttle Service for AHS Debut," IVHS Journal, 2(2), 159-173, 1995.

Tsao, J., (1995b) "Stage definition for AHS deployment and an AHS evolutionary scenario," IVHS Journal, 2(4), 359-382, 1995.

Underwood, S., "Social and institutional considerations in intelligent vehicle-highway systems," Technical Paper 901505, Society of Automotive Engineers, 1990.

Varaiya, P., and Shladover, S.E., Sketch of an IVHS Systems Architecture, PATH Research Report UCBITS-PRR-91-3, University of California at Berkeley, 1991.

Varaiya, Pravin, "Smart Cars on Smart Roads: Problems of Control," IEEE Transactions on Automatic Control, 38(2), 195-207, 1993.

Ward, J., "Step by Step to an Automated Highway System—And Beyond.” In: (Ioannou, P., ed.) Automated Highway Systems, New York: Plenum Press, 73-91, 1997. 


\section{BIOGRAPHIES}

Philip Koopman is a Visiting Senior Research Engineer at the Institute for Complex Engineered Systems, Carnegie Mellon University. His experiences include working with the NAHSC architecture team, conducting research in embedded system design methods, designing embedded computer systems for industrial applications, and driving submarines for the U.S. Navy. His research interests include affordable dependability, embedded computing, and system design methodology. He holds a Ph.D. in Computer Engineering from Carnegie Mellon. He may be contacted at koopman@cmu.edu or via http://www.cs.cmu.edu/ koopman

Michelle Bayouth is a Research Engineer at the Robotics Institute, Carnegie Mellon University. Her experiences include Space Shuttle trajectory analysis, International Space Station risk management, and Automated Highway System concept development. She holds a B.S. in Aerospace Engineering from the University of Texas at Austin. She may be contacted at mbayouth+@ ri.cmu.edu or via http://www.cs.cmu.edu/ mbayouth 


\section{Captions:}

Figure 1. Platoons can only be implemented once Free Agents are possible, because a whole Platoon must encompass Free Agent functionality.

Figure 2. Multiple Lane capabilities can only be implemented once Single Lane capability is possible, because Multiple Lane capability adds lane changing onto Single Lane capability.

Figure 3. Some degree of obstacle exclusion is required in any system in order to achieve efficiency. An enhanced capability of obstacle detection augments obstacle exclusion.

Figure 4. Vigilant capabilities can only be implemented once Trusting capabilities are in place.

Figure 5. Capabilities can have two discrete values: a zero bit represents a baseline capability, while a one bit represents an enhanced capability.

Figure 6. The squares in a K-map are a two-dimensional representation of a torus, in which the top wraps around to meet the bottom, and the sides wrap around to meet each other. Each square in a K-map has four neighbors that differ in value by exactly one bit.

Figure 7. A K-map represents potential AHS states, and two possible deployment paths from the baseline capability configuration (0000) to the maximally enhanced configuration (1111).

Figure 8. Alternate deployment paths may be represented as different transition sequences on the deployment map. Some squares may be considered non-viable, such as deploying a Trusting, Multiple Lane system represented by the $X$ marks in this example.

Table I. A binary number can be assigned to any combination of capability enhancements. A " 0 " represents a baseline capability, while a " 1 " indicates an enhanced capability. 


\begin{tabular}{l|llll}
$\begin{array}{l}\text { BINARY } \\
\text { NUMB }\end{array}$ & $\begin{array}{l}\text { ENTITY SIZE } \\
\text { (first bit) }\end{array}$ & $\begin{array}{l}\text { NUMBER OF } \\
\text { AUTOMATED LANES } \\
\text { (second bit) }\end{array}$ & $\begin{array}{l}\text { OBSTACLE } \\
\text { STRATE GY } \\
\text { (third bit) }\end{array}$ & $\begin{array}{l}\text { SYSTEM VIG ILANCE } \\
\text { (fo urth bit) }\end{array}$ \\
\hline $\mathbf{0 0 0 0}$ & FREE AGENT & SINGLE LANE & EXCLUSION & TRUSTING \\
$\mathbf{0 0 0 1}$ & FREE AGENT & SINGLE LANE & EXCLUSION & VIGILANT \\
$\mathbf{0 0 1 0}$ & FREE AGENT & SINGLE LANE & DETECTION & TRUSTING \\
$\mathbf{0 0 1 1}$ & FREE AGENT & SINGLE LANE & DETECTION & VIGILANT \\
$\mathbf{0 1 0 0}$ & FREE AGENT & MULTIPLE LANES & EXCLUSION & TRUSTING \\
$\mathbf{0 1 0 1}$ & FREE AGENT & MULTIPLE LANES & EXCLUSION & VIGILANT \\
$\mathbf{0 1 1 0}$ & FREE AGENT & MULTIPLE LANES & DETECTION & TRUSTING \\
$\mathbf{0 1 1 1}$ & FREE AGENT & MULTIPLE LANES & DETECTION & VIGILANT \\
$\mathbf{1 0 0 0}$ & PLATOON & SINGLE LANE & EXCLUSION & TRUSTING \\
$\mathbf{1 0 0 1}$ & PLATOON & SINGLE LANE & EXCLUSION & VIGILANT \\
$\mathbf{1 0 1 0}$ & PLATOON & SINGLE LANE & DETECTION & TRUSTING \\
$\mathbf{1 0 1 1}$ & PLATOON & SINGLE LANE & DETECTION & VIGILANT \\
$\mathbf{1 1 0 0}$ & PLATOON & MULTIPLE LANES & EXCLUSION & TRUSTING \\
$\mathbf{1 1 0 1}$ & PLATOON & MULTIPLE LANES & EXCLUSION & VIGILANT \\
$\mathbf{1 1 1 0}$ & PLATOON & MULTIPLE LANES & DETECTION & TRUSTING \\
$\mathbf{1 1 1 1}$ & PLATOON & MULTIPLE LANES & DETECTION & VIGILANT
\end{tabular}

Koopman \& Bayouth -- Table I. 